5th International Workshop on Astronomy and

Relativistic Astrophysics (IWARA2011)

International Journal of Modern Physics: Conference Series

Vol. 18 (2012) 178-181

(C) World Scientific Publishing Company

DOI: $10.1142 / \mathrm{S} 2010194512008410$

\title{
C I 5380 Å: A SPECTRAL LINE WITH LOW INFLUENCE FROM STARSPOTS DYNAMICS AND MAGNETIC CYCLES
}

\author{
D. SOUTO \\ Departamento de Física, Universidade Federal do Rio Grande do Norte, \\ 59072-970 Natal, RN., Brazil \\ dsouto@dfte.ufrn.br \\ J. D. DO NASCIMENTO JR. \\ Departamento de Física, Universidade Federal do Rio Grande do Norte, \\ 59072-970 Natal, RN., Brazil \\ dias@dfte.ufrn.br
}

\begin{abstract}
In the Sun-as-a-star Project, the sun was observed spectroscopically and photometrically for more than 25 years in order to determine variability and luminosity changes. This project detected systematic longterm decrease in the total irradiance as a consequence of the solar magnetic activity cycle (scale of years) and variability on solar activity from a time scale of days-months. The solar magnetic activity cycles could mimic the radial velocity modulation signal of a long-period companion in several spectral lines. This effect is an important limitation for the exoplanet searches programs using the radial velocity technique. The Lomb-Scargle periodogram analysis of the Sun-as-a-star spectroscopic data shows that the photospheric line C I $5380 \AA$ and other 11 lines seems to not show significant influence from the rotational or cromospheric magnetic activity modulation. Thus, our analysis suggest that C I $5380 \AA$ line could be used in programs that require extremely line stability.
\end{abstract}

Keywords: Activity; Line formations; Radial velocity method.

\section{Introduction}

Since 1978 photospheric lines C I $5380 \AA$ have been monitored on the Sun from the Sun-as-a-star Project and their line depths and equivalent widths measured, both full-disk (Gray \& Livingston 1997) and at center-disk (Livingston \& Wallace 2003). The legacy of the Sun-as-a-star Project showed that most solar indices such as 10cm flux, Ca K index, sunspot number, etc. represent, in one way or another, the presence of surface magnetic field. The characteristics of these lines, their response to any temperature changes, and the active region effects on them, have been analyzed in a series of papers (Penza et al. 2004, Penza et al. 2006). In general, changes in Fraunhofer line strength depend in a sensitive way on changes in temperature and pressure in the stellar atmosphere. However, the equivalent widths of some lines appear to be unaffected by the activity magnetic cycle from years scale and from 
days-month scale. On this study we analyzed all photospheric and chromospheric lines from the Sun-as-a-star Project from Livingston et al. (2007). Our main goal is to classify the spectral lines with low influence from starspots dynamics and magnetic cycles.

\section{Observational data}

We use the solar spectrum data derived from the Sun-As-A-star Project and discussed in Livingston et al. (2007). The project collected solar spectra since 1976 until the present day. We searched for detect variations in the spectral line depth over the years of observation. The photometric system of the project and some systematics effects present in the spectra, such as photon noise, scintillation, clouds and collimation effects are described in Brault et al. (1971). Given the high signal to noise generated on the observational span we can consider that the photon noise is negligible $(0.02 \%)$. The analysis of Livingston \& Holweger (1982) shows the ephocs on which the observational data presents corrupted data by the CCD changing and calibrations. For observations between 1976 to 1979 and from 1992 to 1996 the data were not used in the periodogram analyses.

\section{Periodicity analysis}

Since the goal is to find the periods with the analysis of variation in the spectral line depth, we use the formalism developed by Lomb (1976) and Scarlge (1982) and refined by Horne \& Baliunas (1986). The periods identified as significant are those who have had the False Alarm Probabilistic $<10^{-5}$ as discussed Horne \& Baliunas (1986). The error in the measurement was obtained by supposing a Full Width at Half Maximum (FWHM) of the higher peak in the periodogram.

\subsection{Lines with low sensitivity to magnetic activity}

Between the spectral lines analyzed, we found that some spectral lines present low influence from the stellar magnetic activity cycles. Among others, we can point out: Ni I $5220.3 \AA$, O I $7774.2 \AA$, C I $5380.3 \AA$, Cu I $5220.1 \AA$, O I $7771.9 \AA$, Li 6707.8 $\AA$, Ni $6768.0 \AA$, O I $7775.4 \AA$, Fe $6707.4 \AA$, Si I $10827 \AA$, He I $10832 \AA$, He I 10834 $\AA$. In Figure 1 we present the typical behavior for spectral lines with low sensitivity to the magnetic activity cycles. Some of these spectral lines can be useful for the exoplanets search program based on spectroscopic methods.

\section{Conclusions}

In this study, we analyzed several lines from the Sun-As-A-star Project. Based on Lomb-Scargle procedure we conclude that Ni I $5220.3 \AA$, O I $7774.2 \AA$, C I 5380.3 $\AA$, Cu I $5220.1 \AA$, O I $7771.9 \AA$, Li $6707.8 \AA$, Ni $6768.0 \AA$, O I $7775.4 \AA$, Fe $6707.4 \AA$, 


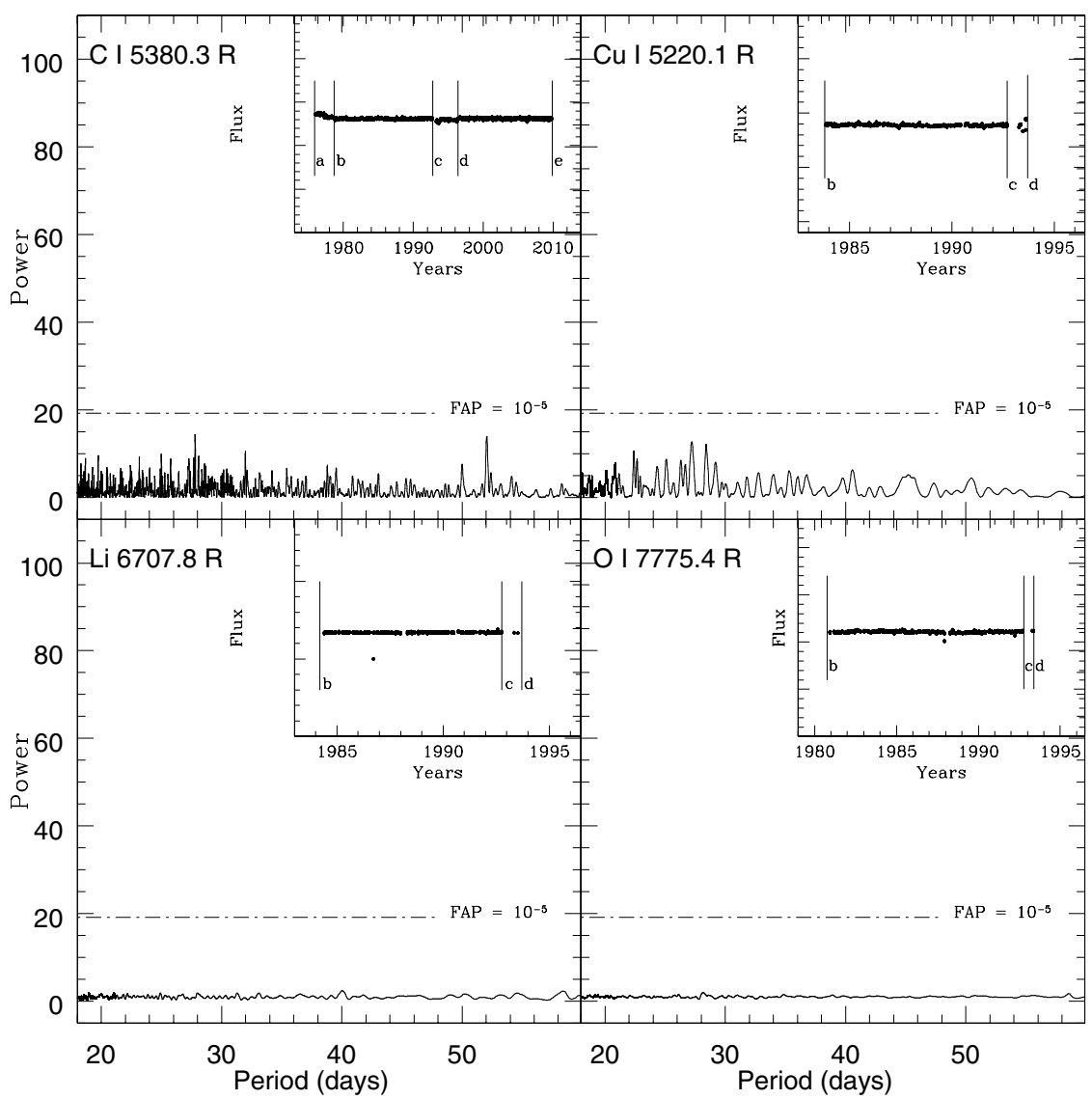

Fig. 1. Lomb-Scargle analysis for the spectral lines C I $5380.3 \AA$, Cu I $5220.1 \AA$, Li $6707.8 \AA \mathrm{e}$ O I $7775.4 \AA$. On top of each frame we present the signal used on the present analysis.

Si I $10827 \AA$, He I $10832 \AA$, He I $10834 \AA$ are very stable and present low sensitivity to magnetic effects from the stellar activity cycle. These results are particularly important for planet search programs based on doppler spectroscopy.

\section{References}

1. Gray, David F.; Livingston, William C., Astrophysical Journal, v.474, p.802, (1997).

2. Livingston, W.; Wallace, L., Solar Physics, v.212, p.227-237, (2003).

3. Penza, V.; Caccin, B.; Del Moro, D., Astronomy and Astrophysics, v.427, p.345-351, (2004).

4. Penza, V.; Pietropaolo, E.; Livingston, W., Astronomy and Astrophysics, v.454, p.349358, (2006). 
5. Livingston, W.; Wallace, L.; White, O. R.; Giampapa, M. S., Astrophysical Journal v.657, p.1137-1149, (2007).

6. Brault, J. W.; White, O. R., Astronomy and Astrophysics, v.13, p.169, (1971).

7. Livingston, W.; Holweger, H., Astrophysical Journal, v.252, p.375-385, (1982).

8. Lomb, N. R., Astrophysics and Space Science, v.39, p.447-462, (1976).

9. Scargle, J. D., Astrophysical Journal v.263, p.835-853, (1982).

10. Horne, J. H.; Baliunas, S. L., Astrophysical Journal, v.302, p.757-763, (1986). 